\title{
Déclaration du Réseau des laboratoires de santé publique du Canada sur les tests sérologiques aux points d'intervention dans le cadre de la COVID-19
}

\author{
Groupe de travail sur les infections par les virus respiratoires ${ }^{1}$
}

\author{
Citation proposée : Groupe de travail sur les infections par les virus respiratoires. Déclaration du Réseau des \\ laboratoires de santé publique du Canada sur les tests sérologiques aux points d'intervention dans le cadre de la \\ COVID-19. Relevé des maladies transmissibles au Canada 2022;48(1):1-3. \\ https://doi.org/10.14745/ccdr.v48i01a01f \\ Mots-clés : COVID-19, test sérologique, point d'intervention, Canada, anticorps contre le SRAS-CoV-2
}

\section{Introduction}

Les tests sérologiques au point d'intervention pour le coronavirus du syndrome respiratoire aigu sévère 2 (SRAS-CoV-2), le virus responsable de la maladie à coronavirus 2019 (COVID-19), détectent la réaction des anticorps humains à l'infection ou à la vaccination et non au virus lui-même. La plupart sont des tests qualitatifs basés sur l'immunochromatographie (flux latéral) qui détectent les lgG+/-lgM dans un échantillon de sang prélevé au doigt et dont les résultats sont disponibles en moins de 30 minutes. Bien que l'adoption de tests sérologiques aux points d'intervention pour la COVID-19 suscite un intérêt général, cette méthode de dépistage présente actuellement des limites importantes, notamment une compréhension incomplète de la réaction immunologique à la COVID-19, des données de validation clinique sous-optimales, une corrélation incertaine (ou I'absence de corrélation) avec les tests sérologiques de laboratoire clinique et une grande variabilité du rendement entre les différents tests aux points d'intervention. La plupart des points clés décrits ci-dessous s'appliquent également aux tests sérologiques COVID-19 en laboratoire.

\section{Position actuelle sur le diagnostic d'infections aiguës}

Les tests sérologiques aux points d'intervention pour la COVID-19 ne sont pas recommandés comme outil de diagnostic de l'infection aiguë et seuls trois produits sont approuvés par Santé Canada à ce jour. En général, ces tests ne sont pas en mesure de détecter les anticorps avant au moins une semaine ou plus après le début des symptômes, et ne sont donc pas adaptés au diagnostic de l'infection aiguë par le SRAS-CoV-2 à ce stade. Nous recommandons que la détection des acides nucléiques (e.g. l'amplification en chaîne par polymérase en temps réel) demeure le test de première ligne pour le diagnostic de l'infection aiguë par le SRAS-CoV-2, comme le conseille l'Organisation mondiale de la santé (1).

\section{Points clés}

- Il faut au moins 7 à 14 jours, parfois plus, après l'apparition des symptômes pour que les anticorps se forment. Par conséquent, le dépistage sérologique au point d'intervention tôt dans l'infection peut aboutir à un faux négatif à la COVID-19 à un moment où le malade est le plus infectieux (i.e. qu'un résultat négatif n'exclut pas l'infection).

- Des faux négatifs peuvent se produire chez les personnes âgées et les malades immunodéprimés, qui ne peuvent réagir en produisant suffisamment d'anticorps.

- Comme les tests sérologiques aux points d'intervention ne détectent pas le virus, leur résultat, qu'il soit positif ou négatif, ne détermine pas si une personne est infectieuse.

- Les résultats positifs peuvent être dus à une infection passée ou récente par le SRAS-CoV-2 ou à la vaccination contre la COVID-19. 
- La plupart des tests sérologiques aux points d'intervention ne font pas la différence entre les anticorps sécrétés lors d'une infection antérieure et ceux générés en réaction à la vaccination contre la COVID-19. Compte tenu de la progression rapide de la vaccination contre la COVID-19, cela limite encore plus I'usage des tests sérologiques aux points d'intervention.

- Comme pour les autres plateformes sérologiques COVID-19, on peut obtenir de faux positifs si ces trousses présentent une réaction croisée avec des anticorps provenant d'une exposition récente ou passée à d'autres coronavirus, $y$ compris les coronavirus humains.

- D'autres infections, ainsi que des états non infectieux (e.g. maladies à facteur rhumatoïde positif), peuvent aussi produire de faux positifs.

- Les faux positifs sont plus probables dans les régions à faible prévalence et à faible vaccination. L'épidémiologie locale et la probabilité prétest de l'individu (i.e. les facteurs de risque cliniques et épidémiologiques) doivent être prises en compte à l'interprétation des résultats de la sérologie aux points d'intervention.

- L'interprétation de la plupart des tests sérologiques aux points d'intervention étant visuelle, elle peut produire de faux positifs et des faux négatifs si elle est inexacte ou subjective.

- Conformément aux recommandations du Comité consultatif national de l'immunisation, il n'y a pas d'indication pour la sérologie avant ou après la vaccination contre la COVID-19.

- Les trousses doivent faire l'objet d'une évaluation approfondie de leurs caractéristiques de rendement (sensibilité, spécificité) avant d'être utilisées en milieu clinique, y compris sur le terrain.

On peut envisager un usage évolutif et exceptionnel des tests sérologiques aux points d'intervention si ces tests ne sont pas disponibles en laboratoire ou s'ils sont trop lents pour repérer les patients COVID-19 les plus susceptibles de bénéficier d'un traitement par anticorps monoclonaux anti-SRAS-CoV-2. Les tests sérologiques ont actuellement une utilité clinique limitée; cependant, certaines compétences ont recommandé leur usage pour éclairer le traitement des patients atteints de la COVID-19, car les premières données des essais cliniques ont montré que certains traitements par anticorps monoclonaux (e.g. casirivimab + imdevimab) donnaient les meilleurs résultats chez les patients séronégatifs. Même dans ce contexte, nous recommandons que les tests sérologiques aux points d'intervention soient réalisés dans un laboratoire afin d'atténuer certains risques décrits ci-dessus et pour qu'ils soient validés avant d'être utilisés comme cela est décrit ci-dessous. Lorsque cela est possible, il est préférable d'effectuer un dépistage sérologique du SRAS-CoV-2 en laboratoire.

\section{Position actuelle sur l'usage en tant que " certificats ou passeports immunitaires »}

La détection des anticorps comme preuve d'immunité pour faciliter la circulation des personnes dans les zones publiques et pour permettre les voyages internationaux fait l'objet d'un débat permanent. Les connaissances sur l'immunité contre le SRAS-CoV-2 évoluent rapidement; cependant, à I'heure actuelle, les corrélats de la protection et la durée de l'immunité ne sont pas bien compris. Par conséquent, nous ne recommandons pas la sérologie, y compris les tests aux points d'intervention, pour déterminer l'immunité individuelle ou pour établir des exemptions aux mesures de santé publique.

\section{Points clés}

- Comme il n'existe actuellement aucun corrélat de protection, on ignore si les niveaux d'anticorps détectés par les tests sérologiques aux points d'intervention sont suffisants pour assurer une protection.

- Comme les tests aux points d'intervention ne fournissent pas de résultat quantitatif, leur utilité peut être limitée même lorsqu'un corrélat de protection est établi.

- Les anticorps contre la COVID-19 persistent au moins six mois; cependant, la vitesse à laquelle les anticorps diminuent varie en fonction de l'âge, de l'immunité de la personne et de la gravité de la maladie.

- Les anticorps de liaison détectés par les tests sérologiques aux points d'intervention ne correspondent pas forcément aux anticorps neutralisants (i.e. protecteurs).

- Étant donné qu'il faut au moins 7 à 14 jours (plus longtemps chez certaines personnes) pour qu'une réaction à anticorps se mette en place, un résultat négatif n'exclut pas l'existence d'une infection active ni l'infectiosité; il ne signifie donc pas que la personne ne peut pas transmettre le SRAS-CoV-2. Les tests sérologiques ne doivent pas remplacer les tests moléculaires (ou antigéniques) pour les voyages ou d'autres types de dépistage.

- Bien que la réinfection ou l'infection après vaccination soit relativement rare, un résultat sérologique positif ne garantit pas la protection contre l'infection, surtout en cas d'expositions intenses et d'émergence de variantes du SRAS-CoV-2 pouvant échapper au système immunitaire.

- Étant donné que les tests sérologiques aux points d'intervention ne détectent pas l'immunité à médiation cellulaire T contre le SRAS-CoV-2, qui est également importante pour la protection à long terme, un résultat négatif ne prouve pas que l'individu n'est pas immunisé.

- La modélisation a montré que des mesures de santé publique, telles que le port du masque et la distanciation physique, seront nécessaires pour contrôler la propagation du SRAS-CoV-2 jusqu'à ce que la population soit 
suffisamment vaccinée et bien immunisée. Ainsi, un résultat sérologique positif, y compris par un test au point d'intervention, peut donner à l'individu un faux sentiment de protection contre l'infection par le SRAS-CoV-2.

\section{Considérations importantes dans le dépistage aux points d'intervention}

Le rôle de la sérologie dans le diagnostic de l'infection par le SRAS-CoV-2, la prise en charge des patients, et les tests d'immunité sont d'une utilité limitée. Une fois que la dynamique de la réaction sérologique à la COVID-19 sera mieux comprise et qu'un corrélat de protection sera trouvé, la sérologie pourra jouer un rôle important dans les mesures de santé publique relatives à la population. Si le dépistage sérologique au point d'intervention est mis en œuvre dans un but précis (e.g. pour le traitement par anticorps monoclonaux), les points suivants doivent être pris en compte :

- Validation approfondie du ou des tests par rapport au " gold standard " (tests de neutralisation virale ou autre test sérologique de laboratoire). Les caractéristiques de rendement (sensibilité, spécificité, valeurs prédictives positives et négatives, réaction croisée avec d'autres coronavirus) doivent être établies à partir de sérums de patients infectés par le SRAS-CoV-2 (ancestral et variants), d'autres virus respiratoires, y compris les coronavirus saisonniers, et de témoins sains.

- Fournir une formation adéquate au personnel de santé et de laboratoire pour réaliser le test et interpréter le résultat.

- Réalisation d'une évaluation du risque d'infection par le SRAS-CoV-2 et la transmission d'infections hématogènes pour l'opérateur. Nous recommandons l'application de mesures universelles pour prévenir la transmission d'agents pathogènes par voie hématogène (au minimum, des gants et des blouses) lors du dépistage aux points d'intervention jusqu'à ce que le risque pour l'opérateur puisse être évalué en bonne et due forme.

- Établir un programme permanent de contrôle et d'assurance de la qualité avant la mise en œuvre.

- Prendre des dispositions pour la saisie des données relatives aux tests dans les dossiers des malades et à des fins de surveillance, et établir l'obligation de participer à une évaluation externe de la qualité pour maintenir celle-ci à un niveau élevé.
S'appuyant sur les données disponibles, le Réseau des laboratoires de santé publique du Canada recommande de ne pas recourir pour l'instant aux tests sérologiques COVID-19 aux points d'intervention pour les tests cliniques ou d'immunité ordinaires. Conformément aux recommandations du Comité consultatif national de l'immunisation (2), les tests sérologiques ne doivent pas être utilisés pour documenter le statut vaccinal ou pour évaluer la réaction à la vaccination contre la COVID-19. Au fur et à mesure que l'on dispose de plus de données sur les corrélats immunologiques de la protection, la durée de l'immunité, le rendement des tests, et que les tests sont validés par rapport aux méthodes sérologiques de référence, l'application clinique des tests aux points d'intervention sera réévaluée. Les tests moléculaires, tels que l'amplification en chaîne par polymérase en temps réel, restent la principale méthode de dépistage en laboratoire de l'infection aiguë par le SRAS-CoV-2 et de diagnostic de la COVID-19.

\section{Références}

1. World Health Organization. Advice on the use of point-ofcare immunodiagnostic tests for COVID-19. Switzerland (CH): WHO; April 2020. https://www.who.int/news-room/ commentaries/detail/advice-on-the-use-of-point-of-careimmunodiagnostic-tests-for-covid-19

2. Agence de la santé publique du Canada. Comité consultatif national de l'immunisation. Recommandations sur I'utilisation des vaccins contre la COVID-19. https://www. canada.ca/fr/sante-publique/services/immunisation/comiteconsultatif-national-immunisation-ccni/recommandationsutilisation-vaccins-covid-19.html\#a76 